\title{
An Extended Modified Fuzzy Possibilistic C-Means Clustering Algorithm for Intrusion Detection
}

\author{
Sarab M. Hameed, Sumaya Saad, and Mayyadah F. AlAni
}

\begin{abstract}
Computer intruders have become a major threat due to their wide spread through the Internet. Therefore, there was a need for security technique that monitors computer resources and sends reports on the activities of any anomaly or strange patterns which called Intrusion Detection (ID). A fuzzy clustering algorithm used because the boundaries between normal and intrusive cannot be well distinct due to the uncertainty nature of an attack. This paper proposed an algorithm for ID that combines both Modified fuzzy possiblistic C-Means (MFPCM) and symbolic fuzzy clustering in one algorithm called Extended Modified Fuzzy Possiblistic C-means (EMFPCM). To evaluate the EMFPCM, Knowledge Discovery and Data Mining Cup 1999 (KDD cup 99) intrusion detection dataset was used. The results indicated that the proposed algorithm was able to distinguish between normal and attack behaviors with high detection rate.
\end{abstract}

Index Terms-Fuzzy clustering, modified fuzzy possibilistic C-mean, intrusion detection, mixed features, symbolic data.

\section{INTRODUCTION}

Intrusion Detection (ID) has been defined as "the problem of identifying individuals who are using a computer system without authorization and those who have legitimate access to the system but are abusing their privileges" [1]. Identification is done by analyzing the events occurring in a computer system or network then search for signs of intrusions that compromise the confidentiality, integrity, availability, or to bypass the security mechanisms of a computer or network [2].

ID uses cluster analysis as a technique for classifying data. Cluster analysis classifies network packets into normal or intrusion. In classical cluster analysis each datum must be assigned to exactly one cluster. This requirement is alleviates by fuzzy cluster analysis by allowing gradual memberships. This offers dealing with data that belong to more than one cluster at the same time [3].

Clustering is found to be the widely used approach in intrusion detection system (IDS). Compared with the clustering algorithms, the fuzzy approaches are found to be efficient. Dunn in 1974 was first introduced Fuzzy C-Means clustering model (FCM) then Bezdek in 1983 extended and generalized this model [4]. Since then, different improvements of the method and model are suggested by researchers.

Reference [5] presents a clustering algorithm which uses

Manuscript received February 20, 2013; revised April 25, 2013.

The authors are with the Computer Science Department, University of Baghdad, Jadiriya, P.O.Box 17635, Iraq (e-mail: sarab_majeed@ scbaghdad.edu.iq, sumasaad@yahoo.com,mfaisal@uob.edu.bh). fuzzy connectedness as the similarity metric for intrusion detection. Experimental results showed the stability of efficiency and accuracy of the algorithm.

Toosi and Kahani in reference [6] proposed five neuro-fuzzy classifiers for IDS, each for classifying data from one class in the Knowledge Discovery in Database (KDD99) dataset. Initial classification was performed using a set of parallel neuro-fuzzy classifiers. Then according to the output, the fuzzy inference system makes final decision on the current behavior as normal or intrusion. The structure of neuro-fuzzy engine optimized using genetic algorithm. The system problem was the great time consuming.

Reference [7] presents intrusion detection system based on C-fuzzy decision tree. The algorithm enhanced the performance of IDSs and emphasized the importance of modified tree in developing improved IDSs.

Whereas the researchers of reference [8] evaluated three methods for symbolic features transformation including: indicator variables, conditional probabilities and the separability split value method. All of these methods were compared with the arbitrary conversion method and applied to an ID problem and the KDDCup99 dataset. The results showed that the prediction capability of the classifiers utilized was improved with these three transformation methods.

Jawhar and Mehrotra in reference [9] proposed an intrusion detection model based on hybrid fuzzy logic and neural network. The first stage of this model was applying FCM to classify the data into two clusters normal and attack. The second stage used MLP for classification of attacks. The number of hidden layers and its number of nodes was determined experimentally. This model has ability to recognize an attack, to differentiate one attack from another and to detect new attacks with high detection rate and low false negative. Meanwhile, reference [10] shows a new approach, called FC-ANN, based on artificial neural network and fuzzy clustering, to solve the problem and help IDS achieve higher detection rate, less false positive rate and stronger stability.

In addition, the authors of reference [11] proposed a two-phase intrusion detection algorithm. Weighted fuzzy clustering was done based on the statistical information according to proposed weighted fuzzy cluster feature. The number of clusters for fuzzy clustering could be changed dynamically. Theoretical analysis and experimental results showed the algorithm can detect the intrusion behaviors effectively.

In this paper an algorithm for intrusion detection that combines both Modified Fuzzy Possiblistic C-Means (MFPCM) and fuzzy clustering for symbolic features in one 
algorithm proposed to distinguish between normal and attack behaviors.

The following sections of the paper are organized as follows: Section II gives background on MFPCM clustering algorithm and symbolic fuzzy clustering. Section III describes the proposed intrusion detection algorithm. Section IV presents the description of Knowledge Discovery and Data Mining Cup 1999 (KDD cup 99) on which the proposed algorithm experiments are conducted. Section V illustrates the results obtained from implementing and testing the proposed algorithm. Finally, conclusion is presented in Section VI.

\section{MFPCM CLUSTERING ALGORITHM}

A number of fuzzy clustering methods have been developed following the general fuzzy set theory strategies outlined by Zadeh [12]. Optimal clustering is determined by most fuzzy clustering algorithms based on minimizing objective function. Each cluster is represented by a cluster prototype. The prototype consists of a cluster center additional information about the size and the shape of the cluster may be included [13].

The key to the success of the cluster analysis is the choice of an appropriate objective function to obtain better quality clustering results. MFPCM aims to give good results relating to the original FPCM algorithm by adding new weight of data points in relation to every cluster and modifying the exponent of the distance between a point and a class [14],[15].

MFPCM objective function $\left(J_{m f p c m}\right)$ is formulated in equation (1) [14].

$$
\begin{gathered}
J_{m f p c m}=\sum_{i=1}^{c} \sum_{k=1}^{n}\left(\mu_{i k}^{m} w_{k i}^{m} d^{2 m}\left(x_{k}, v\right)+\right. \\
{ }_{\left.\left.t_{i k} w_{k i} w_{k i}^{\delta} d^{2 \delta}\left(x_{k}, v_{i}\right)\right)\right)}
\end{gathered}
$$

where

$n$ is the number of patterns in the dataset.

$c$ is the number of clusters.

$m$ is any real number in the range $1 \leq m \prec \infty$.

$\delta$ is any real number in the range $1 \leq \delta \prec \infty$.

$\mu_{i k}$ is the membership degree of the pattern $x_{k}$ in cluster.

$t_{i k}$ is the possibility degree of the pattern $x_{k}$ in cluster $i$.

$w_{k i}$ is weight of the point $\mathrm{k}$ in relation to the class $i$.

$x_{k}$ is the kth pattern.

$v_{i}$ is the center (prototype) of the ith cluster.

And $d^{2}\left(x_{k}, v_{i}\right)$ is dissimilarity measure between the pattern $x_{k}$ and the center $v_{i}$ of specific cluster $i$. The objective function in (1) is optimized through iterative fuzzy partitioning that update of membership $\mu_{i k}$, typicality $t_{i k}$, weights $w_{k i}$ and the cluster centers $v_{i}$ by equations in (2) through (5) [14] .

$$
\begin{gathered}
\mu_{i k}=\left[\sum_{j=1}^{c}\left(\frac{d^{2}\left(x_{k}, v_{i}\right)}{d^{2}\left(x_{k}, v_{j}\right)}\right)^{\frac{2 m}{(m-1)}}\right]^{-1} \\
t_{i k}=\left[\sum_{j=1}^{n}\left(\frac{d^{2}\left(x_{k}, v_{i}\right)}{d^{2}\left(x_{k}, v_{j}\right)}\right)^{\frac{2 \delta}{(\delta-1)}}\right]^{-1} \\
w_{k i}=\exp \left(-\frac{d^{2}\left(x_{k}, v_{i}\right)}{\left(\sum_{j=i}^{n} d^{2}\left(x_{j},-\right)\right) * \frac{c}{n}}\right]^{-}
\end{gathered}
$$

where $\bar{v}=\frac{\sum_{i=1}^{c} v_{i}}{c}$

$$
v_{i}=\frac{\sum_{k=1}^{n}\left(\mu_{i k}^{m} w_{k i}^{m}+t_{i k}^{\delta} w_{k i}^{\delta}\right) x_{k}}{\sum_{k=1}^{n}\left(\mu_{i k}^{m} w_{k i}^{m}+t_{i k}^{\delta} w_{k i}^{\delta}\right)}, 1 \leq i \leq c
$$

The iterations will stop when $\left|\mu_{i k}{ }^{t+1}-\mu_{i k}{ }^{t}\right| \leq \varepsilon$, where $\varepsilon$ a termination criterion between 0 and 1 and $t$ is the iteration step. This procedure converges to a local minimum or a saddle point of $J_{m f p c m}$.

In 1998, El-Sonbaty and Ismail suggested a fuzzy symbolic c-means (SFCM) algorithm [16]. A cluster center is assumed to be formed as a group of features and each feature is composed of several events. Let $v_{j p \mid i}$ be the event of feature $j$ in cluster $i$ and let $e_{j p \mid i}$ be the membership degree of association of the pth event $v_{j p \mid i}$ to the feature $j$ in cluster $i$. Thus, the $j$ th feature of the ith cluster center $v_{i j}$ can be presented as equation (6) [17].

$$
v_{i j}=\left[\left(v_{j 1 \mid i}, e_{j 1 \mid i}\right), \ldots,\left(v_{j p \mid i}, e_{j p \mid i}\right)\right]
$$

where

$$
e_{j p \mid i}=\frac{\sum_{k=1}^{n}\left(\mu_{i k}\right)^{m} \theta}{\sum_{k=1}^{n}\left(\mu_{i k}\right)^{m}}
$$

where $\theta \in\{0,1\}$ and $\theta=1$ if the $j$ th feature of the $k$ th datum $x_{k}$ consists of the pth event, otherwise $\theta=0$. $\mu$ is the membership degree $\mu_{i k}$ of each pattern $k$ in cluster $i$ and the member degree is updated according to (2).

\section{THE PROPOSED EMFPCM FOR ID}

The ID problem is viewed in signature network-based model as a two-class clustering problem: that classify patterns in normal and intrusive categories.

Extended Modified Fuzzy Possiblistic C-(EMFPCM) algorithm is proposed as a hybrid algorithm inspired from 
MFPCM and SFCM to deal with the uncertainty nature of attacks to manipulate network traffic patterns that contains symbolic features in addition to the numeric features.

The objective function of the EMFPCM is the same as MFPCM objective function shown in (1) except the distance which is adjusting by a new proposed equation. The proposed equation calculates the distance as shown in (8).

$$
d^{2}\left(x_{k}, v_{i}\right)=\sum_{s=1}^{\mathrm{sf}} d_{s}\left(x_{k}, v_{i}\right)+d_{N}\left(x_{k}, v_{i}\right)
$$

where

$s f$ is the number of symbolic features.

$d_{s}\left(x_{k}, v_{i}\right)$ represents the similarity metric for symbolic features in the network traffic data stream and it is computed with proposed formula shown in (9).

$$
d_{s}\left(x_{k}, v_{i}\right)=\sum_{p=1}^{e n}\left(\text { difference }_{i k} \times e_{i}^{p}\right)
$$

where

$e n$ is the number of events for feature $s$.

$$
\text { difference }_{i k} \begin{cases}0 & \text { if } x_{k}=v_{i}^{p} \\ 1 & \text { otherwise }\end{cases}
$$

$d_{N}\left(x_{k}, v_{i}\right)$ is the Euclidean distance that is used to measure the similarity among numeric features in the network traffic data stream.

Moreover, the (5) which computes the centroid for symbolic features are tuned as in (11) to deal with both numeric and symbolic features by inserting $\beta$ condition to new centroid computation equation.

$$
v_{i}=\frac{\sum_{k=1}^{n}\left(\mu_{i k}^{m} w_{k i}^{m}+t_{i k}^{\delta} w_{k i}^{m}\right) \beta}{\sum_{k=1}^{n}\left(\mu_{i k}^{m} w_{k i}^{m}+t_{i k}^{\delta} w_{k i}^{m}\right)}
$$

where

$n$ number of patterns.

$\mu_{i k}$ is calculated according to (2)

$t_{i k}$ is computed according to (3)

$w_{k i}$ is computed as in (4)

Fuzziness control $(m)$, with $m>1$

Possibilistic parameter $(\delta)$, with $\delta>1$

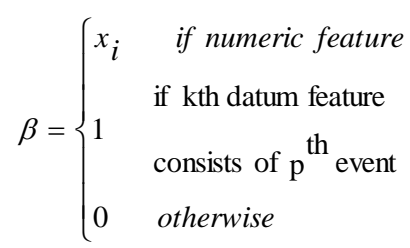

The proposed algorithm (EMFPCM) can be outlined in the following steps.

\section{Setp1: Initialization}

Number of clusters $c=2$.

Number of patterns in the dataset $(n)$.

Number of features $(n f)$.
Fuzziness control $(m, \eta)$, with $(m, \eta)>1$.

Iteration number $t=0$.

Stopping criterion $\varepsilon=0.001$.

Initialize centroids $\left(v_{i}{ }^{(0)}\right)$

Step2: Calculation

For $1 \leq k \leq n$

For $1 \leq i \leq c$

1) Compute the distance $d^{2}\left(x_{k}, v_{i}\right)$ between the $k$ th pattern and ith the cluster center using (8).

2) Update the fuzzy membership degree $\mu_{i k}^{(t)}$ using (2).

3) Update the fuzzy typicality degree $t_{i k}^{(t)}$ using (3).

4) Update the weight $w_{i k}^{(t)}$ using (5).

5) Compute the cluster center $v_{i}^{(t)}$ using (11)

Step3: Stopping criterion

If $\max _{i k}\left\{\left|v_{i k}^{t+1}-v_{i k}^{t}\right|\right\} \prec \varepsilon$ then STOP

Else Increment iteration number $t=t+1$ and GO TO step 2.

\section{DATASET DESCRIPTION}

The experiments were carried out on a real data stream called intrusion detection dataset called KDD cup 99 which has been used in as benchmar k for security researches [18]. KDD cup 99 dataset was derived in 1999 from the DARPA98 network traffic dataset by assembling individual TCP packets into TCP connections. The KDD cup 99 dataset includes a set of 41 features derived for each connection and a label which specifies the status of connection records as either normal or specific attack type.

The attacks fall in one of the following four categories [19]:

1) Denial of Service (DoS): Attacker tries to prevent legitimate users from using a service.

2) Remote to Local (R2L): Attacker does not have an account on the victim machine, hence tries to gain access.

3) User to Root (U2R): Attacker has local access to the victim machine and tries to gain super user privileges.

4) Probe: Attacker tries to gain information about the target host.

Table I shows multiple types of attack while Table II shows the three components of KDD dataset.

TABLE I: ATTACK TYPES [KDD99]

\begin{tabular}{ll}
\hline \hline Category & Type \\
\hline DoS & smurf, neptune, back, teardrop, pod, land \\
Probe & satan, ipsweep, portsweep, nmap \\
R2L & warezclient, guess_passwd, warezmaster, ftp_write, \\
& $\begin{array}{l}\text { multihop, phf, spy, imap } \\
\text { buffer_overflow,rootkit, loadmodule, perl }\end{array}$ \\
\hline
\end{tabular}

TABLE II: THE ARRANGEMENT OF CHANNELS

\begin{tabular}{llllll}
\hline \hline KDD dataset & DoS & Probe & R2L & U2R & Normal \\
\hline Whole & 3883370 & 41102 & 1126 & 52 & 972780 \\
Corrected & 229853 & 4166 & 16347 & 70 & 60593 \\
$10 \%$ & 391458 & 4107 & 1126 & 52 & 97277 \\
\hline \hline
\end{tabular}




\section{EXPERIMENTAL RESULTS}

The network traffic data from the KDD Cup 99 dataset was used to evaluate the proposed algorithm. The following subsections illustrate the KDD Cup 99 dataset preprocessing, the performance evaluation and results of the EMFPCM.

\section{A. KDD Cup 99 Preparation and Features Selection}

Features in the KDD cup 99 dataset covers different forms including: continuous and symbolic [18]. The numeric features in KDD99 have different scales and this causes bias over some feature over the others for this reason a normalization process was required. The normalization process applied on numeric features as follows:

1) Features with small scale were scaled linearly to the range $[0.0,1.0]$ using the formula (13).

$$
\bar{X}=\frac{X-\operatorname{Min} X}{\operatorname{Max} X-\operatorname{Min} X}
$$

where $X$ is the numerical attribute value, $\operatorname{Min} X$ is the minimum value that the attribute $X$ can get and $\operatorname{Max} X$ is the maximum value that the attribute $X$ can get.

2) Features with large scale including src_bytes $[0$, 1.3billion] and dest_bytes [0, 1.3billion] features, were normalized by applying the logarithmic scaling (with base 10) [19].

3) Boolean features whose value was either 0 or 1 and features whose value in the range $[0.0,1.0]$ are not normalized.

As mentioned in Section IV, there are 41 features in KDD cup 99 dataset. The proposed algorithm used 30 features by eliminating the features that have little significant in intrusion detection, to do this an approach based on information gain was employed [20]. Based on the entropy of a feature, information gain measures the relevance of a given feature, i.e. measures its role in determining the class label. If the feature is relevant then the calculated entropies will be close to 0 and the information gain will be close to 1 . Information gain was calculated for discrete features, continuous features should be discretized. To this, continuous features were partitioned into partitions depending on its instances frequency, i.e. the feature space was partitioned into arbitrary number of partitions where each partition contains $N$ dataset instances of the feature value [21].

Let $S$ be a set of training set samples with their corresponding labels. Suppose there are $\mathrm{m}$ classes, the training set contains $s_{i}$ samples of class $I$ and $s$ is the total number of samples in the training set. Expected information needed to classify a given sample was calculated by [21]:

$$
I\left(s_{1}, s_{2}, \ldots . ., s_{m}\right)=-\sum_{i=1} \frac{s_{i}}{s} \log _{2}\left(\frac{s_{i}}{s}\right)
$$

A feature $F$ with values $\{f 1, f 2, \ldots, f v\}$ can divide the training set into $v$ subsets $\{S 1, S 2, \ldots, S v\}$ where $S j$ was the subset which has the value $f j$ for feature $F$. Furthermore let $S j$ contain $s_{i j}$ samples of class $i$. Entropy of the feature $F$ was:

$$
E(F)=\sum \frac{s_{1 j}+\ldots+s_{m j}}{s} * I\left(s_{1 j}, \ldots ., s_{m j}\right)
$$

Information gain for $F$ can be calculated as:

$$
\operatorname{Gain}(F)=I\left(s_{1}, \ldots ., s_{m}\right)-E(F)
$$

In our experiments, information gain was calculated for each feature. The features with negative values discarded including features: land, wrong_fragment, urgent, num-compromised, su_attempted, num_root, num_shell, num-outbound_cmds, is_host_login, and is_guset login as shown in Table III (the shaded texts represent the excluded features). Elimination of these features will increase the speed of the proposed algorithm without affecting accuracy.

TABLE III: DATA TYPES FEATURES

\begin{tabular}{l|l}
\hline Types & Features \\
\hline Continues & duration, src_bytes, dst_bytes, wrong_fragment, urgent,hot, \\
& num_failed_logins, logged_in, num_compromised, \\
& root_shell, su_attempted, num_root, num_file_ \\
creations,num_shells, num_access_files, \\
num_outbound_cmds, count, srv_count, serror_rate, \\
& srv_serror_rate, rerror_rate, srv_rerror_rate, same_srv_rate, \\
& diff_srv_rate, srv_diff_host_rate, dst_host_count, \\
& dst_host_srv_count, dst_host_same_srv_rate, \\
& dst_host_diff_srv_rate, dst_host_same_src_port_rate, \\
& dst_host_srv_diff_host_rate, dst_host_serror_rate, \\
& dst_host_srv_serror_rate, dst_host_rerror_rate,dst_ \\
& host_srv_rerror_rate. \\
protocol_type, service, flag, land, logged_in, is_host_login, & is_guest_login.
\end{tabular}

\section{B. Performance Evaluation}

ID effectiveness was evaluated by its capability to make accurate predictions. According to the real nature of a given event compared to the prediction from the ID, four possible outcomes are shown in Table IV, known as the confusion matrix [22].

TABLE IV: CONFUSION MATRIX

\begin{tabular}{lcc}
\hline \hline Actual Class & Predicted Class & Predicted Class \\
& Negative class (Normal) & Positive class (Attack) \\
\hline Normal & True Negative (TN) & False positive (FP) \\
Attack & False Negative (FN) & True Positive (TP) \\
\hline \hline
\end{tabular}

where True negatives (TN) as well as true positives (TP) correspond to a correct operation of the IDS that is, events are successfully labeled as normal and attacks, respectively.

False positives (FP) refer to normal events being predicted as attacks; false negatives (FN) are attack events incorrectly predicted as normal events [21]. A high FP rate will seriously affect the performance of the system being detected. A high FN rate will leave the system vulnerable to intrusions. So, both FP and FN rates should be minimized, together with maximizing TP and $\mathrm{TN}$ rates.

In view of that, to evaluate the performance of the proposed algorithm for ID, detection rate (i.e. the proportion of true positives which are correctly identified as such), false alarm rate (i.e. the proportion of all negative substances that are incorrectly identified as positive), and accuracy (i.e. the proportion of true results in the population) were calculated separately as in equations 17,18 , and 19 respectively [22]. 


$$
\begin{gathered}
D R=\frac{T P}{T P+F N} \\
F A R=\frac{F P}{T N+F P} \\
\text { Accuracy }=\frac{T P+T N}{T P+T N+F N+F P}
\end{gathered}
$$

\section{Results}

The whole data set is huge, so only $10 \%$ subset is used to create a smaller training and testing set in order to evaluate the effectiveness of EMPFCM algorithm. First, for initialization step one normal pattern was selected randomly to initialize the center of the first cluster and four attack patterns were selected randomly to initialize the center of the second cluster.

Then in training phase the EMFPCM was training using 4000 randomly selected normal and attacks patterns from " $10 \%$ KDD" dataset to tune the cluster centroid of the proposed algorithm (i.e., generate normal and attack signatures).

Finally, in testing phase, 5552 instances data of KDD cup 99 passed through the trained model to detect the intrusions and computed the detection rate (DR), false alarm (FAR), and accuracy of EMFPCM.

The proposed EMFPCM was tested with instances containing 41 and 30 features in two experiments. The results of the proposed algorithm were compared with traditional MFPCM as in Table V. In these two experiments, 2776 patterns were selected randomly from " $10 \% \mathrm{KDD}$ " dataset.

In the first experiment (EXP1), the selected sample contained normal and the most common attacks patterns namely smurf, ipsweep, neptune and back, most attacks could be distinguished from the normal activities and the average DR was as high as $99.05 \%$. At the same time, the percentage of normal events that were incorrectly labeled as attachs (i.e. FAR) was 1.6. Whereas, in the second experiment (Exp2) which conducted to test the ability of EMFPCM to detect new variations of attacks, sample contained different attacks that did not exist in the training dataset. The DR is $99.7 \%$, which is better than before, while the FAR was slightly different. EMFPCM results improved the DR which is important in most applications and FAR nearly the same with all kind of attacks.

TABLE V: EXPERIMENTAL RESULTS OF THE EMFPCM ALGORITHM

\begin{tabular}{llccc}
\hline \hline & Metric & MFPCM & $\begin{array}{c}\text { EMFPCM with } \\
\text { 41 Features }\end{array}$ & $\begin{array}{l}\text { EMFPCM with } \\
\text { 30 Features }\end{array}$ \\
\hline EXP1 & DR & 98.51 & 98.94 & 99.05 \\
& FAR & 1.6 & 1.66 & 1.6 \\
& Accuracy & 98.35 & 98.78 & 98.89 \\
EXP2 & DR & 99.22 & 99.6 & 99.71 \\
& FAR & 1.1 & 1.12 & 1.1 \\
& Accuracy & 99.11 & 99.5 & 99.61 \\
\hline \hline
\end{tabular}

\section{CONCLUSIONS}

An EMFPCM with mixed features clustering algorithm was proposed for intrusion detection problem. This algorithm used KDD cup 99 benchmark intrusion detection dataset for training and testing.

The EMFPCM algorithm used 30 features with optimal sensitivity and highest discriminatory power to speed of the algorithm without affecting accuracy. Also, the EMFPCM algorithm provided better result than conventional MFPCM and the average DR of the proposed algorithm was 99.7 which was outperform the average DR of classical algorithm which was 99.2.

\section{REFERENCES}

[1] B. Mukherjee, L. T. Heberlein, and K. N. Levitt, "Network Intrusion Detection," IEEE Network, vol. 8, no. 3, pp. 26-41, May/June 1994.

[2] R. Bace and P. Mell, Intrusion Detection Systems, NIST Special Publications SP 800-31, 2001.

[3] H. Timm and R. Kruse, "A Modification to Improve Possibilistic Fuzzy Cluster Analysis," in Proc. the 2002 IEEE International Conf. on Fuzzy Systems, 2002, vol. 2, pp. 1460-1465.

[4] J. C. Bezdek, R. Ehrlich, and W. Full, "FCM: The Fuzzy C-Means Clustering Algorithm," Computers \& Geosciences, vol. 10, no. 2-3, pp. 191-203, 1984.

[5] Q. Wang and V. Megalooikonomou, "A Clustering Algorithm for Intrusion Detection," in Proc. the SPIE Conf. on Data Mining, Intrusion Detection, Information Assurance, and Data Networks Security, Florida, 2005, pp. 31-38.

[6] A. N. Toosi and M. Kahani, "A Novel Soft Computing Model Using Adaptive Neuro-Fuzzy Inference System for Intrusion Detection," in Proc. the 2007 IEEE International Conf. on Networking, Sensing and Control, London, 2007, pp. 15-17.

[7] K. Makkithaya, N. Reddy, and U. D. Acharya, "Intrusion Detection System using Modified C-Fuzzy Decision Tree Classifier," International Journal of Computer Science and Network Security, vol. 8, no. 11, pp. 29-35, November 2008.

[8] E. Hernandez-Pereira, J. A. Suarez-Romero, O. Fontenla-Romero, and A. Alonso-Betanzos, "Conversion Methods for Symbolic Features: A Comparison Applied to an Intrusion Detection Problem," Expert Systems with Applications International journal, vol. 36, no. 7, pp. 10612-10617, 2009.

[9] M. Mehrotra and M. Jawhar, "Design Network Intrusion Detection System Using Hybrid Fuzzy-Neural Network," International Journal of Computer Science and Security, vol. 4, no. 3, pp. 285-294, 2010.

[10] G. Wang, J. Hao, J. Ma, and L. Huang, "A New Approach to Intrusion Detection Using Artificial Neural Networks and Fuzzy Clustering," Expert Systems with Applications Journal, vol. 37, no. 9, pp. 6225-6232, 2010.

[11] S. Xiao-Ke, L. Yang, Q. Yu-Ming, W. Ren-Xia, and C. Yao-Dong, "Two-phase Intrusion Detection Algorithm in Mixed Attributes Data Stream," Computer Engineering Journal, vol. 36, no. 18, pp. 19-20, 2010.

[12] S. Nascimento, B. Mirkin, and F. Moura-Pires, "A Fuzzy Clustering Model of Data and Fuzzy c-Means," in Proc. the 9th IEEE International Conf. on Fuzzy Systems, San Antonio, 2000, vol. 1, pp. 302-307.

[13] H. Timm, C. Borgelt, and R. Kruse, "An Extension of Possibilistic Fuzzy Cluster Analysis,” Fuzzy Sets and Systems, vol. 147, no. 1, pp. 3-16, May 2004.

[14] M. Saad and A. Alimi, "Modified Fuzzy Possibilistic C-means," in Proc. the International MultiConf. of Engineers and Computer Scientists, March 2009, vol. 1, pp. 18-20.

[15] D. Vanisri and C. Loganathan, "An Efficient Fuzzy Possibilistic C-Means with Penalized and Compensated Constraints," Global Journal of Computer Science and Technology, vol. 11, no. 1, pp. 15-21, March 2011

[16] Y. El-Sonbaty and M. A. Ismail, "Fuzzy Clustering for Symbolic Data," IEEE Trans. Fuzzy Systems vol. 6, no. 3, pp. 195-204, 1998.

[17] M.-S. Yang, P.-Y. Hwang, and D.-H. Chen, "Fuzzy Clustering Algorithms for Mixed Feature Variables," Elsevier, Fuzzy Sets and Systems, vol. 141, no. 2, pp. 301-317, January, 2004.

[18] KDD-CUP 1999 Dataset. [Online]. Avialable: http://kdd.ics.uci.edu/databases/.

[19] B. Mukherjee and T. Ramesh, "Network Intrusion Detection System using Reduced Dimensionality," Indian Journal of Computer Science and Engineering, vol. 2 ,no. 1, pp. 61-67, 2011.

[20] M. Sabhnani and G. Serpent, "Application of Machine Learning Algorithms to KDD Intrusion Detection Dataset within Misuse Detection Context," in Proc the International Conf. on Machine 
Learning: Models, Technologies, and Applications, Las Vegas, vol. 1, pp. 209-215, 2003.

[21] H. Kayacık, A. Zincir-Heywood, and I. M. Heywood, "Selecting Features for Intrusion Detection: A Feature Relevance Analysis on KDD 99 Intrusion Detection Datasets," in Proc. the 3rd Annu. Conf. on Privacy Security and Trust, 2005, pp. 3-8.

[22] S. X. Wu and W. Banzhaf, "The Use of Computational Intelligence in Intrusion Detection Systems: A Review," Journal of Applied Soft Computing Journal, Elsevier, vol. 10, no. 1, pp. 1-35, January 2010.

Sarab M. Hameed was born in Baghdad, Iraq in 1971. She received the B.Sc. and M.Sc. degrees in computer science from University of Baghdad, Iraq in 1992 and 1999, respectively, and the Ph.D. degree in computer science from University of Technology, Iraq in 2005. She is now an associate professor in the Department of Computer Science at the University of Baghdad. Her research areas mainly include network security, Cryptography, Steganography.

Dr. Sarab M. Hameed joins different committees at Ministry, University, College and Department levels. She is a member in scientific and examination committees at College and Department levels.

Sumaya Saad was born in Baghdad, Iraq in 1983. She received the B.Sc. and M.Sc. degrees in computer science from University of Baghdad, Iraq in
2005 and 2013, respectively. She is now a lecturer in the Department of Computer Science at the University of Baghdad.

Sumaya Saad research areas mainly include network security. She joins different committees at College and Department levels.

Mayyadah F. AlAni was born in Baghdad, Iraq 1968. She received her B.Sc. and M.Sc. degrees in computer science from University of Baghdad, Iraq in 1989 and 1996, respectively. She received her Ph.D. degree in computer science from University of Technology, Iraq in 2002.

She joined Department of Computer Science/ University of Baghdad in September 1989. She supervised research thesis students in image processing and evolutionary algorithms. She is now working with Department of Computer Science/ University of Bahrain since 2005 teaching different courses for graduate and undergraduate students.

She is an associate professor and she has more than 25 publications. Her main research interest is in computer vision, evolutionary algorithms security and e-learning.

Dr. Mayyadah Alani is joined different committees at University, College and Department levels. She is a member in scientific research committees at University, College and Department. She is the chairperson of Senior Project Committee and the coordinator of reviewing exam paper. In addition, she is a member in Department ABET committee. 